\title{
Computational and Numerical Solutions for $(2+1)$-Dimensional Integrable Schwarz-Korteweg-de Vries Equation with Miura Transform
}

\author{
Raghda A. M. Attia,, ${ }^{1,2}$ S. H. Alfalqi, ${ }^{3}$ J. F. Alzaidi, ${ }^{3}$ Mostafa M. A. Khater $\mathbb{D}^{1,4}$ \\ and Dianchen $\mathrm{Lu} \mathrm{DiD}^{1}$ \\ ${ }^{1}$ Department of Mathematics, Faculty of Science, Jiangsu University, Zhenjiang 212013, China \\ ${ }^{2}$ Department of Basic Science, Higher Technological Institute, 10th of Ramadan 44634, Egypt \\ ${ }^{3}$ Department of Mathematics, Faculty of Science and Arts in Mahayil Asir, King Khalid University, Abha, Saudi Arabia \\ ${ }^{4}$ Department of Mathematics, El Obour Institutes, Cairo 11828, Egypt
}

Correspondence should be addressed to Mostafa M. A. Khater; mostafa.khater2024@yahoo.com

Received 3 June 2020; Accepted 23 July 2020; Published 14 September 2020

Academic Editor: Alina Gavriluţ

Copyright (C) 2020 Raghda A. M. Attia et al. This is an open access article distributed under the Creative Commons Attribution License, which permits unrestricted use, distribution, and reproduction in any medium, provided the original work is properly cited.

This paper investigates the analytical, semianalytical, and numerical solutions of the $(2+1)$-dimensional integrable Schwarz-Korteweg-de Vries (SKdV) equation. The extended simplest equation method, the sech-tanh method, the Adomian decomposition method, and cubic spline scheme are employed to obtain distinct formulas of solitary waves that are employed to calculate the initial and boundary conditions. Consequently, the numerical solutions of this model can be investigated. Moreover, their stability properties are also analyzed. The solutions obtained by means of these techniques are compared to unravel relations between them and their characteristics illustrated under the suitable choice of the parameter values.

\section{Introduction}

The Korteweg-de Vries (KdV) equation is a seminal model in fluid mechanics. This model was introduced by Boussinesq in 1877 and reintroduced by Diederik Korteweg and Gustav de Vries in 1895. The KdV has the following formula [1-9]:

$$
Q_{t}+\mathscr{U}_{x x x}-6 \mathcal{U}_{x}=0,
$$

where $\mathcal{U}=\mathscr{U}(x, t)$ characterizes the weakly nonlinear shallow water waves. Equation (1) can be written in many distinct forms and combined with other models. One of them is the Schwarz-Korteweg-de Vries (SKdV) equation given by

$$
\mathbb{Q}_{t}+\mathbb{Q}_{x}\left[\left(\frac{Q_{x x}}{Q_{x}}\right)-\frac{1}{2}\left(\frac{Q_{x x}}{\mathbb{Q}_{x}}\right)^{2}\right]=0
$$

where $Q=Q(x, t)$ is the unknown function. The $\mathrm{SKdV}$ was derived by Krichever and Novikov [10] and Weiss $[11,12]$.

In this paper, we study the $(2+1)$-dimensional integrable generalization of SKdV as follows:

$$
\mathcal{U}_{t}+\frac{1}{4} \mathcal{U}_{x x y}-\frac{\mathscr{U}_{x} \mathcal{U}_{x y}}{2 \mathcal{U}}-\frac{\mathcal{U}_{x x} \mathcal{U}_{y}}{4 \mathcal{U}}+\frac{\mathscr{U}_{x}^{2} \mathcal{U}_{y}}{2 \mathscr{U}^{2}}-\frac{\mathcal{U}_{x}}{8} \int\left(\frac{\mathcal{U}_{x}^{2}}{\mathcal{U}^{2}}\right)_{y} \mathrm{~d} x=0 .
$$

Equation (3) was derived by Toda and $\mathrm{Yu}$ [13]. Using the following transformation on equation (3),

$$
\begin{aligned}
\mathcal{U} & =\mathcal{S}_{x}, \\
\mathcal{S} & =e^{\mathscr{F}}, \\
\mathscr{F}{ }_{t} & =\mathscr{U}, \\
\mathscr{F}_{t} & =\mathscr{R},
\end{aligned}
$$


where $\quad \mathcal{S}=\mathcal{S}(x, t), \mathscr{F}=\mathscr{F}(x, t), \mathcal{U}=\mathscr{U}(x, t), \quad$ and $\mathscr{R}=\mathscr{R}(x, t)$ are the unknown functions, we obtain

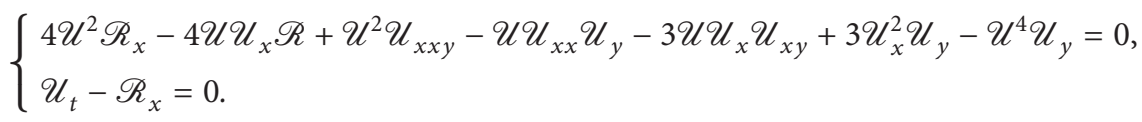

This equation is obtained from the study by Bruzón et al. [14-16]. Using the Miura transform [17-19] on equation (5) as

$$
\begin{aligned}
& \mathscr{B}_{x}=\frac{\mathscr{U}_{x x}}{4 \mathcal{U}}-\frac{3 \mathcal{U}_{x}^{2}}{8 \mathcal{U}^{2}}-\frac{\mathscr{U}^{2}}{8}, \\
& \mathscr{B}_{y}=-\frac{\mathscr{R}}{\mathscr{U}},
\end{aligned}
$$

we obtain $[20,21]$

$$
4 \mathscr{B}_{x t}+\mathscr{B}_{x x x y}+8 \mathscr{B}_{x y} \mathscr{B}_{x}+4 \mathscr{B}_{y} \mathscr{B}_{x x}=0 .
$$

If we adopt the wave transformation

$$
\begin{aligned}
\mathscr{B}(x, y, t) & =\mathscr{B}(\mathfrak{Z}), \\
\mathcal{Z} & =\rho x+\delta y+c t,
\end{aligned}
$$

then we convert equation (7) into an ordinary differential equation (NLODE). The integration of the obtained NLODE with zero constant of integration leads to

$$
4 c \mathscr{B}^{\prime}+\rho^{2} \delta \mathscr{B}^{\prime \prime \prime}+6 \rho \delta \mathscr{B}^{\prime 2}=0 .
$$

If we consider the substitution $\mathscr{B}^{\prime}=\mathscr{F}$, then it results in

$$
4 c \mathscr{F}+\rho^{2} \delta \mathscr{F}^{\prime \prime}+6 \rho \delta \mathscr{F}^{2}=0 .
$$

Having these ideas in mind, this paper is organized as follows: Section 2 presents the two methods and derives the solutions of the SKdV equation. Section 5 represents the solutions for several numerical values of the parameters. Additionally, their stability and properties are also discussed. Finally, Section 6 summarises the main conclusions.

\section{Explicit Solutions}

In this section, we apply two analytical techniques for deriving the solutions of the $(2+1)$-dimensional integrable SKdV model. We adopt the extended simplest equation method and the sech-tanh method to obtain various distinct formulas of solitary wave solutions of equation (3). For further details about the two methods, see [22-26].

2.1. Extended Simplest Equation. According to the homogeneous balance rule between the highest derivative and the nonlinear term in equation (9), we obtain $n=2$. Thus, the general solution of equation (10) is given by

$$
\mathscr{F}(\mathbb{Z})=\sum_{i=-n}^{n} a_{i} \mathscr{C}(\mathbb{Z})^{i}=a_{2} \mathscr{C}(\mathbb{Z})^{2}+a_{1} \mathscr{C}(\mathbb{Z})+\frac{a_{-2}}{\mathscr{C}(3)^{2}}+\frac{a_{-1}}{\mathscr{C}(3)}+a_{0},
$$

where $a_{i}(i=-2, \ldots, 2)$ are arbitrary constants. Additionally, $\mathscr{C}$ (3) satisfies the following ordinary differential equation:

$$
\mathscr{C}^{\prime}(\boldsymbol{Z})=\alpha+\lambda \mathscr{C}(\boldsymbol{Z})+\mu \mathscr{C}(\boldsymbol{Z})^{2},
$$

where $\beta, \alpha$, and $\mu$ are the arbitrary constants. Substituting equations (11) and (12) into (9) and collecting all terms of $\mathscr{C}^{i}$ (3) $(i=-4,-3, \ldots, 3,4)$, we get a system of algebraic equations. Solving this system, we obtain two families of solutions.

Family 1

$$
\begin{aligned}
& a_{0} \longrightarrow \frac{1}{6}\left(-2 \alpha \mu \rho-\lambda^{2} \rho\right), \\
& a_{1} \longrightarrow 0 \\
& a_{2} \longrightarrow 0 \\
& a_{-1} \longrightarrow-\alpha \lambda \rho, \\
& a_{-2} \longrightarrow-\alpha^{2} \rho, \\
& c \longrightarrow \frac{1}{4}\left(\delta \lambda^{2} \rho^{2}-4 \alpha \delta \mu \rho^{2}\right) .
\end{aligned}
$$

Family 2

$$
\begin{aligned}
& a_{0} \longrightarrow \frac{1}{6}\left(-2 \alpha \mu \rho-\lambda^{2} \rho\right), \\
& a_{1} \longrightarrow-\lambda \mu \rho, \\
& a_{2} \longrightarrow-\mu^{2} \rho, \\
& a_{-1} \longrightarrow 0 \\
& a_{-2} \longrightarrow 0 \\
& c \longrightarrow \frac{1}{4}\left(\delta \lambda^{2} \rho^{2}-4 \alpha \delta \mu \rho^{2}\right) .
\end{aligned}
$$

From these two families, the solitary wave solutions of equation (7) can be obtained.

According to Family 1, we have the following expressions. 
2.1.1. When $\lambda=0$. For $\alpha \mu>0$, we obtain

$$
\begin{aligned}
\mathscr{F}_{1}(x, y, t)= & -\frac{\alpha \mu \rho}{3}-\frac{\lambda^{2} \rho}{6}-\alpha \mu \rho \cot ^{2}\left(\frac{1}{4} \sqrt{\alpha \mu}\left(\delta \rho^{2} t\left(\lambda^{2}-4 \alpha \mu\right)+4 \rho x+4 \delta y+4 \vartheta\right)\right) \\
& -\lambda \rho \sqrt{\alpha \mu} \cot \left(\frac{1}{4} \sqrt{\alpha \mu}\left(\delta \rho^{2} t\left(\lambda^{2}-4 \alpha \mu\right)+4 \rho x+4 \delta y+4 \vartheta\right)\right), \\
\mathscr{F}_{2}(x, y, t)= & -\frac{\alpha \mu \rho}{3}-\frac{\lambda^{2} \rho}{6}-\alpha \mu \rho \tan ^{2}\left(\frac{1}{4} \sqrt{\alpha \mu}\left(\delta \rho^{2} t\left(\lambda^{2}-4 \alpha \mu\right)+4 \rho x+4 \delta y+4 \vartheta\right)\right) \\
& -\lambda \rho \sqrt{\alpha \mu} \tan \left(\frac{1}{4} \sqrt{\alpha \mu}\left(\delta \rho^{2} t\left(\lambda^{2}-4 \alpha \mu\right)+4 \rho x+4 \delta y+4 \vartheta\right)\right) .
\end{aligned}
$$

For $\alpha \mu<0$, we obtain

$$
\begin{aligned}
& \mathscr{F}_{3}(x, y, t)=\alpha \mu \rho \operatorname{coth}^{2}\left(\frac{1}{4} \sqrt{-\alpha \mu}(\rho(4 x-4 \alpha \delta \mu \rho t)+4 \delta y) \mp \frac{\log (\vartheta)}{2}\right)-\frac{\alpha \mu \rho}{3}, \\
& \mathscr{F}_{4}(x, y, t)=\alpha \mu \rho \tanh ^{2}\left(\frac{1}{4} \sqrt{-\alpha \mu}(\rho(4 x-4 \alpha \delta \mu \rho t)+4 \delta y) \mp \frac{\log (\vartheta)}{2}\right)-\frac{\alpha \mu \rho}{3} .
\end{aligned}
$$

When $4 \alpha \mu>\lambda^{2}$, we obtain

$$
\begin{aligned}
\mathscr{F}_{5}(x, y, t)= & -\frac{\alpha \mu \rho}{3}-\frac{4 \alpha^{2} \mu^{2} \rho}{\left(\lambda-\sqrt{4 \alpha \mu-\lambda^{2}} \tan \left((1 / 8) \sqrt{4 \alpha \mu-\lambda^{2}}\left(\rho\left(\delta \rho t\left(\lambda^{2}-4 \alpha \mu\right)+4 x\right)+4 \delta y+49\right)\right)\right)^{2}} \\
& -\frac{\lambda^{2} \rho}{6}+\frac{2 \alpha \lambda \mu \rho}{\lambda-\sqrt{4 \alpha \mu-\lambda^{2}} \tan \left((1 / 8) \sqrt{4 \alpha \mu-\lambda^{2}}\left(\rho\left(\delta \rho t\left(\lambda^{2}-4 \alpha \mu\right)+4 x\right)+4 \delta y+4 \vartheta\right)\right)}, \\
\mathscr{F}_{6}(x, y, t)= & -\frac{\alpha \mu \rho}{3}-\frac{4 \alpha^{2} \mu^{2} \rho}{\left(\lambda-\sqrt{4 \alpha \mu-\lambda^{2}} \cot \left((1 / 8) \sqrt{4 \alpha \mu-\lambda^{2}}\left(\rho\left(\delta \rho t\left(\lambda^{2}-4 \alpha \mu\right)+4 x\right)+4 \delta y+4 \vartheta\right)\right)\right)^{2}} \\
& -\frac{\lambda^{2} \rho}{6}+\frac{2 \alpha \lambda \mu \rho}{\lambda-\sqrt{4 \alpha \mu-\lambda^{2}} \cot \left((1 / 8) \sqrt{4 \alpha \mu-\lambda^{2}}\left(\rho\left(\delta \rho t\left(\lambda^{2}-4 \alpha \mu\right)+4 x\right)+4 \delta y+49\right)\right)} .
\end{aligned}
$$


According to Family 2, we have the following 2.1.2. When $\lambda=0$. For $\alpha \mu>0$, we obtain expressions.

$$
\begin{aligned}
\mathscr{F}_{7}(x, y, t)= & -\frac{\alpha \mu \rho}{3}-\frac{\lambda^{2} \rho}{6}-\alpha \mu \rho \tan ^{2}\left(\frac{1}{4} \sqrt{\alpha \mu}\left(\delta \rho^{2} t\left(\lambda^{2}-4 \alpha \mu\right)+4 \rho x+4 \delta y+4 \vartheta\right)\right) \\
& -\lambda \rho \sqrt{\alpha \mu} \tan \left(\frac{1}{4} \sqrt{\alpha \mu}\left(\delta \rho^{2} t\left(\lambda^{2}-4 \alpha \mu\right)+4 \rho x+4 \delta y+4 \vartheta\right)\right), \\
\mathscr{F}_{8}(x, y, t)= & -\frac{\alpha \mu \rho}{3}-\frac{\lambda^{2} \rho}{6}-\alpha \mu \rho \cot ^{2}\left(\frac{1}{4} \sqrt{\alpha \mu}\left(\delta \rho^{2} t\left(\lambda^{2}-4 \alpha \mu\right)+4 \rho x+4 \delta y+4 \vartheta\right)\right) \\
& -\lambda \rho \sqrt{\alpha \mu} \cot \left(\frac{1}{4} \sqrt{\alpha \mu}\left(\delta \rho^{2} t\left(\lambda^{2}-4 \alpha \mu\right)+4 \rho x+4 \delta y+4 \vartheta\right)\right) .
\end{aligned}
$$

For $\alpha \mu<0$, we obtain

$$
\begin{aligned}
& \mathscr{F}_{9}(x, y, t)=\alpha \mu \rho \tanh ^{2}\left(\frac{1}{4} \sqrt{-\alpha \mu}(\rho(4 x-4 \alpha \delta \mu \rho t)+4 \delta y) \mp \frac{\log (\vartheta)}{2}\right)-\frac{\alpha \mu \rho}{3}, \\
& \mathscr{F}_{10}(x, y, t)=\alpha \mu \rho \operatorname{coth}^{2}\left(\frac{1}{4} \sqrt{-\alpha \mu}(\rho(4 x-4 \alpha \delta \mu \rho t)+4 \delta y) \mp \frac{\log (\vartheta)}{2}\right)-\frac{\alpha \mu \rho}{3} .
\end{aligned}
$$

When $\alpha=0$ : For $\lambda>0$, we get

$$
\begin{aligned}
\mathscr{F}_{11}(x, y, t)= & -\frac{\lambda^{2} \mu^{2} \rho \exp \left((1 / 2) \lambda\left(\rho\left(\delta \lambda^{2} \rho t+4 x\right)+4 \delta y+49\right)\right)}{6\left(\mu e^{(1 / 4) \delta \lambda^{3} \rho^{2} t+\lambda \rho x+\delta \lambda y+\lambda \vartheta}-1\right)^{2}} \\
& -\frac{2 \lambda^{2} \mu \rho e^{(1 / 4) \lambda \rho\left(\delta \lambda^{2} \rho t+4 x\right)+\delta \lambda y+\lambda \vartheta}}{3\left(\mu e^{(1 / 4) \delta \lambda^{3} \rho^{2} t+\lambda \rho x+\delta \lambda y+\lambda \vartheta}-1\right)^{2}} \\
& -\frac{\lambda^{2} \rho}{6\left(\mu e^{(1 / 4) \delta \lambda^{3} \rho^{2} t+\lambda \rho x+\delta \lambda y+\lambda \vartheta}-1\right)^{2}} .
\end{aligned}
$$

For $\lambda<0$, we obtain

$$
\begin{aligned}
\mathscr{F}_{12}(x, y, t)= & -\frac{\mu^{4} \rho \exp \left((1 / 2) \lambda\left(\rho\left(\delta \lambda^{2} \rho t+4 x\right)+4 \delta y+4 \vartheta\right)\right)}{\left(\mu e^{(1 / 4) \delta \lambda^{3} \rho^{2} t+\lambda \rho x+\delta \lambda y+\lambda \vartheta}+1\right)^{2}} \\
& -\frac{\lambda^{2} \rho}{6\left(\mu e^{(1 / 4) \delta \lambda^{3} \rho^{2} t+\lambda \rho x+\delta \lambda y+\lambda \vartheta}+1\right)^{2}} \\
& -\frac{\lambda^{2} \mu^{2} \rho \exp \left((1 / 2) \lambda\left(\rho\left(\delta \lambda^{2} \rho t+4 x\right)+4 \delta y+4 \vartheta\right)\right)}{6\left(\mu e^{(1 / 4) \delta \lambda^{3} \rho^{2} t+\lambda \rho x+\delta \lambda y+\lambda \vartheta}+1\right)^{2}} \\
& +\frac{\lambda \mu^{2} \rho e^{1 / 4 \lambda \rho\left(\delta \lambda^{2} \rho t+4 x\right)+\delta \lambda y+\lambda \vartheta}}{\left(\mu e^{(1 / 4) \delta \lambda^{3} \rho^{2} t+\lambda \rho x+\delta \lambda y+\lambda \vartheta}+1\right)^{2}} \\
& -\frac{\lambda^{2} \mu \rho e^{(1 / 4) \lambda \rho\left(\delta \lambda^{2} \rho t+4 x\right)+\delta \lambda y+\lambda \vartheta}}{3\left(\mu e^{(1 / 4) \delta \lambda^{3} \rho^{2} t+\lambda \rho x+\delta \lambda y+\lambda \vartheta}+1\right)^{2}} \\
& +\frac{\lambda \mu^{3} \rho \exp \left(1 / 2 \lambda\left(\rho\left(\delta \lambda^{2} \rho t+4 x\right)+4 \delta y+49\right)\right)}{\left(\mu e^{(1 / 4) \delta \lambda^{3} \rho^{2} t+\lambda \rho x+\delta \lambda y+\lambda \vartheta}+1\right)^{2}} .
\end{aligned}
$$

When $4 \alpha \mu>\lambda^{2}$, we obtain

$$
\begin{aligned}
& \mathscr{F}_{13}(x, y, t)=\frac{2 \alpha \mu \rho}{3}-\frac{\lambda^{2} \rho}{6}+\frac{1}{4} \lambda^{2} \rho \sec ^{2} \\
& \left(\frac{1}{8} \sqrt{4 \alpha \mu-\lambda^{2}}\left(\delta \rho^{2} t\left(\lambda^{2}-4 \alpha \mu\right)+4 \rho x+4 \delta y+49\right)\right) \\
& -\alpha \mu \rho \sec ^{2}\left(\frac{1}{8} \sqrt{4 \alpha \mu-\lambda^{2}}\left(\delta \rho^{2} t\left(\lambda^{2}-4 \alpha \mu\right)+4 \rho x+4 \delta y+49\right)\right),
\end{aligned}
$$

$$
\begin{aligned}
& \mathscr{F}_{14}(x, y, t)=\frac{2 \alpha \mu \rho}{3}-\frac{\lambda^{2} \rho}{6}+\frac{1}{4} \lambda^{2} \rho \csc ^{2} \\
& \left(\frac{1}{8} \sqrt{4 \alpha \mu-\lambda^{2}}\left(\delta \rho^{2} t\left(\lambda^{2}-4 \alpha \mu\right)+4 \rho x+4 \delta y+4 \vartheta\right)\right) \\
& -\alpha \mu \rho \csc ^{2}\left(\frac{1}{8} \sqrt{4 \alpha \mu-\lambda^{2}}\left(\delta \rho^{2} t\left(\lambda^{2}-4 \alpha \mu\right)+4 \rho x+4 \delta y+4 \vartheta\right)\right) .
\end{aligned}
$$

2.2. Sech-Tanh Method. The general solution of equation (10) according to the sech-tanh method and calculated value of balance is given by

$$
\begin{aligned}
\mathscr{F}(3)= & \sum_{i=1}^{n} \operatorname{sech}^{i-1}(3)\left(a_{i} \operatorname{sech}(3)+b_{i} \tanh (3)\right)+a_{0} \\
= & \operatorname{sech}(3)\left(a_{2} \operatorname{sech}(3)+b_{2} \tanh (3)\right) \\
& +a_{1} \operatorname{sech}(3)+a_{0}+b_{1} \tanh (3),
\end{aligned}
$$

where $a_{0}, a_{1}, a_{2}, b_{1}$, and $b_{2}$ are the arbitrary constants. Substituting equation (29) into (10) and collecting all terms of $\operatorname{sech}(3), \operatorname{sech}^{2}(3), \operatorname{sech}^{3}(3), \tanh (3), \tanh (3) \sec h^{2}$ (3), and $\tanh (\xi) \operatorname{sech}(3)$, we obtain a system of algebraic equations. Solving this system, we obtain 


$$
\begin{aligned}
& a_{0} \longrightarrow \frac{2 \sqrt{c}}{3 \sqrt{\delta}}, \\
& a_{1} \longrightarrow 0, \\
& a_{2} \longrightarrow-\frac{\sqrt{c}}{\sqrt{\delta}}, \\
& b_{1} \longrightarrow 0, \\
& b_{2} \longrightarrow 0, \\
& \rho \longrightarrow-\frac{\sqrt{c}}{\sqrt{\delta}}, \quad \text { where }(c>0, \delta>0) .
\end{aligned}
$$

Consequently, the explicit solution of equation (7) is given by

$$
\mathscr{F}_{15}(x, y, t)=\frac{\sqrt{c}\left(2-3 \operatorname{sech}^{2}(c t+\rho x+\delta y)\right)}{3 \sqrt{\delta}} .
$$

\section{Stability Investigation}

We now examine the stability property for $(2+1)$-dimensional integrable SKdV model with the Miura transformation by means of an Hamiltonian system. The momentum $\mathfrak{H}$ in the Hamiltonian system is given by

$$
\mathfrak{H}=\frac{1}{2} \int_{-\mathscr{J}}^{\mathscr{J}} \mathscr{B}^{2}(\Im) \mathrm{d} \mathfrak{Z}
$$

where $\mathscr{B}(\mathcal{Z})$ is the solution of the model. Consequently, the condition for stability of the solutions can be formulated as

$$
\frac{\partial \mathfrak{H}}{\partial c}>0
$$

where $c$ is the wave velocity. The momentum in the Hamiltonian system for equations (18) and (31) are given, respectively, by

$$
\begin{aligned}
\mathfrak{H}= & \frac{1}{c}\left(3200 c-\operatorname{sech}^{2}(10 c+26)-\operatorname{sech}^{2}(10 c+34)+\operatorname{sech}^{2}(26-10 c)+\operatorname{sech}^{2}(34-10 c)-4 \log \left(\left(e^{52}-1\right)\right.\right. \\
& \left.\times \sinh (10 c)+\left(1+e^{52}\right) \cosh (10 c)\right)-4 \log \left(\left(e^{68}-1\right) \sinh (10 c)+\left(1+e^{68}\right) \cosh (10 c)\right)+4 \log \left(\left(1+e^{52}\right) \cosh (10 c)\right. \\
& \left.\left.-\left(e^{52}-1\right) \sinh (10 c)\right)+4 \log \left(\left(1+e^{68}\right) \cosh (10 c)-\left(e^{68}-1\right) \sinh (10 c)\right)\right) \\
\mathfrak{H}= & \frac{1}{72}\left(4\left(100 c+\log \left(e^{7-5 c}+e^{5 c}\right)+\log \left(e^{23-5 c}+e^{5 c}\right)-\log \left(e^{-5 c}+e^{5 c+7}\right)-\log \left(e^{-5 c}+e^{5 c+23}\right)\right)\right. \\
& \left.-\operatorname{sech}^{2}\left(5 c+\frac{7}{2}\right)-\operatorname{sech}^{2}\left(5 c+\frac{23}{2}\right)+\operatorname{sech}^{2}\left(\frac{7}{2}-5 c\right)+\operatorname{sech}^{2}\left(\frac{23}{2}-5 c\right)\right) .
\end{aligned}
$$

And thus,

$$
\begin{gathered}
\left.\frac{\partial \mathfrak{S}}{\partial c}\right|_{c=72}=2.37146 \times 10^{-298}>0, \\
\left.\frac{\partial \mathfrak{S}}{\partial c}\right|_{c=9}=5.55556>0 .
\end{gathered}
$$

We conclude that this solution is stable on the interval $x \in[-5,5], t \in[-5,5]$. This result shows the ability of the solutions for their application. Using the same steps, we can check the stability property of all other obtained solutions.

\section{Semianalytical and Numerical Solutions}

This section applies semianalytical and numerical schemes for deriving the solutions of the $(2+1)$-dimensional integrable SKdV model. The Adomian decomposition method and cubic b-spline schemes are employed to the method to investigate the accuracy of the obtained analytical solutions. Also, this study aims to give a comparison between both used analytical schemes. For further details about the two methods, see [27-30].
4.1. Adomian Decomposition Method. Applying this scheme gives equation (10) in the following form:

$$
\sum_{j=0}^{\infty} \mathscr{F}_{j}(\boldsymbol{Z})=\mathscr{F}(0)+\mathscr{F}^{\prime}(0) \mathcal{Z}-\frac{4 c}{\rho^{2} \delta} \mathscr{L}^{-1}\left(\sum_{j=0}^{\infty} \mathscr{F}_{j}(\mathbb{Z})\right)-\frac{6}{\rho} \mathscr{L}^{-1}\left(\sum_{j=0}^{\infty} A_{j}\right) .
$$

Thus, with respect to equation (18) and the following conditions $\alpha=-1, a_{0}=4, a_{-1}=0, a_{-2}=-3, c=72, \delta=2$, $\lambda=0, \mu=4$, and $\rho=3$, we obtain

$$
\begin{aligned}
& \mathscr{F}_{0}=-2, \\
& \mathscr{F}_{1}=12 \mathfrak{Z}^{2}, \\
& \mathscr{F}_{2}=-8 \mathfrak{J}^{4}, \\
& \mathscr{F}_{3}=8 \mathfrak{Z}^{4}-\frac{16 \mathfrak{Z}^{6}}{3} .
\end{aligned}
$$

Consequently, the semianalytical solution of equation (10) is written in the following form: 


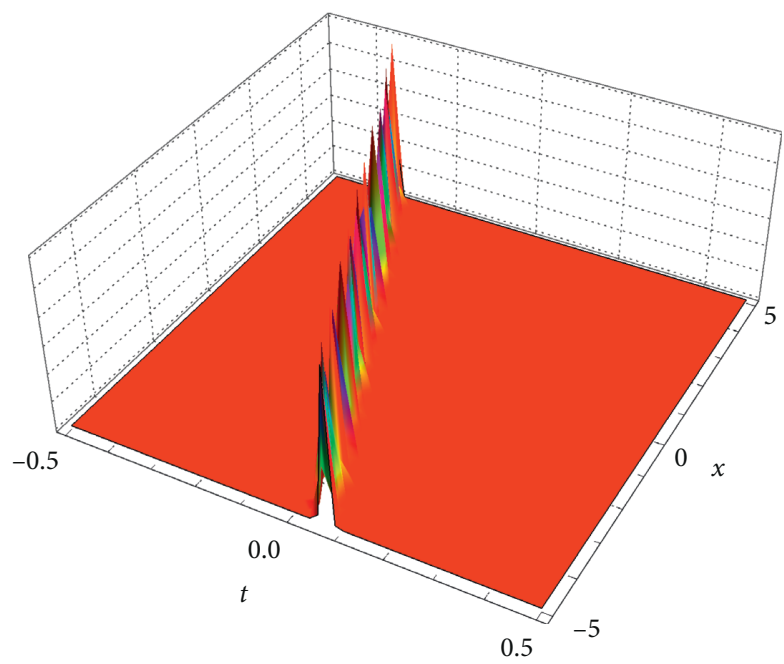

(a)

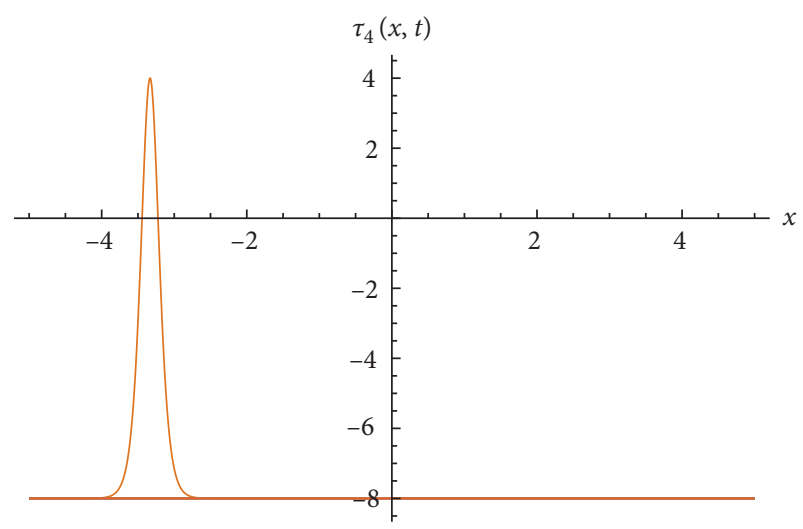

(b)

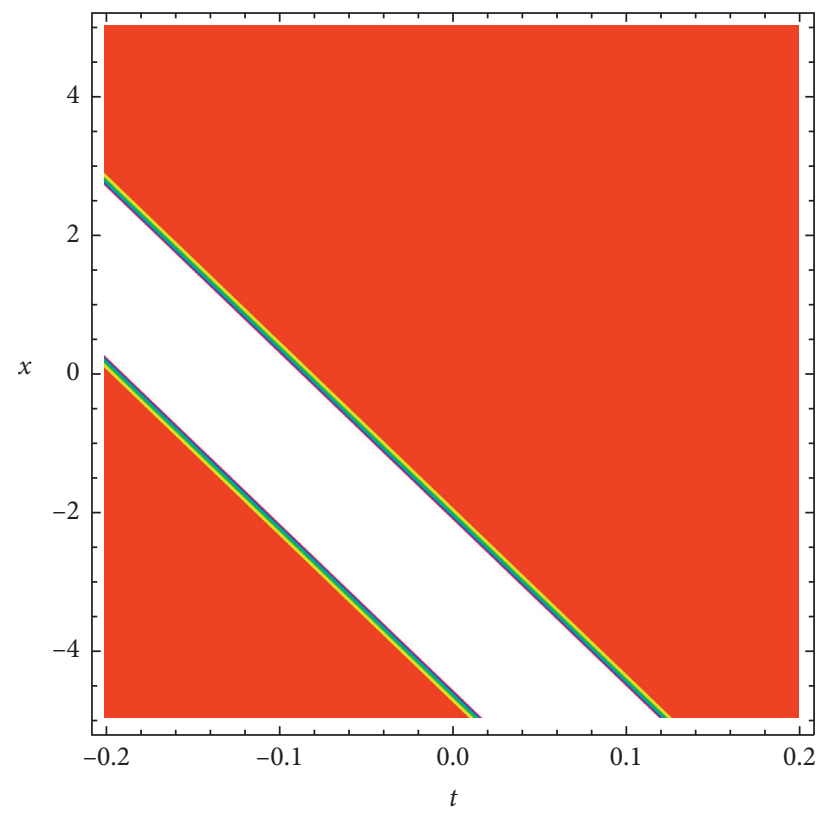

(c)

Figure 1: Solitary wave in three different forms of equation (18).

$$
\mathscr{F}=-\frac{16 \mathfrak{Z}^{6}}{3}+12 \mathfrak{Z}^{2}-2+\cdots
$$

However, with respect to equation (31) and the following conditions $a_{0}=1, a_{1}=0, a_{2}=-(3 / 2), b_{1}=0, b_{2}=0, c=9$, $\delta=4$, and $\rho=-(3 / 2)$, we obtain

$$
\begin{aligned}
& \mathscr{F}_{0}=-\frac{1}{2}, \\
& \mathscr{F}_{1}=\frac{3 \mathfrak{Z}^{2}}{2}, \\
& \mathscr{F}_{2}=-\mathfrak{Z}^{4}, \\
& \mathscr{F}_{3}=\frac{133^{6}}{30}-\frac{\mathfrak{Z}^{4}}{2} .
\end{aligned}
$$

Consequently, the semianalytical solution of equation (10) is written in the following form:

$$
\mathscr{F}=\frac{13 \mathfrak{Z}^{6}}{30}-\frac{3 \mathfrak{Z}^{4}}{2}+\frac{3 \mathfrak{Z}^{2}}{2}-\frac{1}{2}+\cdots
$$

4.2. Cubic B-Spline Scheme. Employing the cubic B-spline scheme to evaluate the numerical solutions of equation (10). Using same initial and boundary conditions with respect to the obtained solutions (18) and (31), yields

\section{Discussion}

This section illustrates several of the results for $\mathscr{F}(x, y, t)$ to highlight the properties of the $(2+1)$-dimensional integrable SKdV model with Miura transformation. In the 


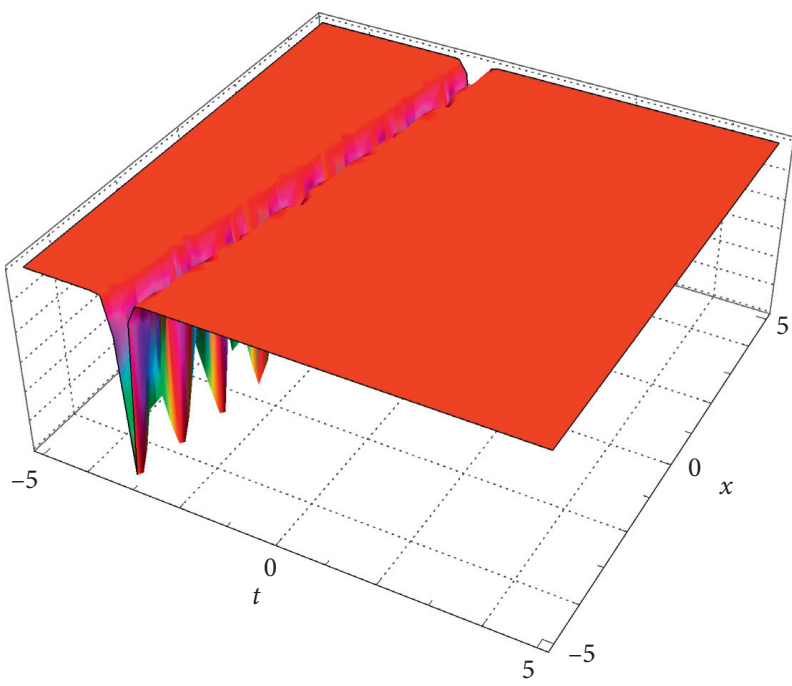

(a)

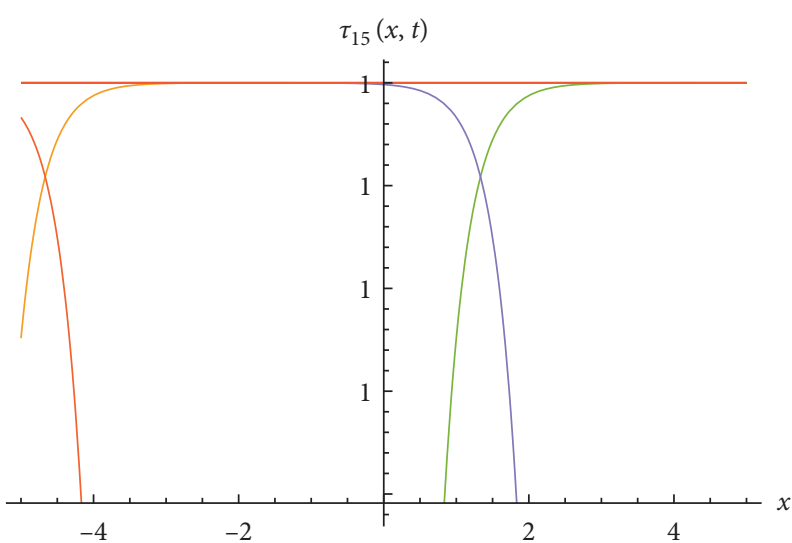

(b)

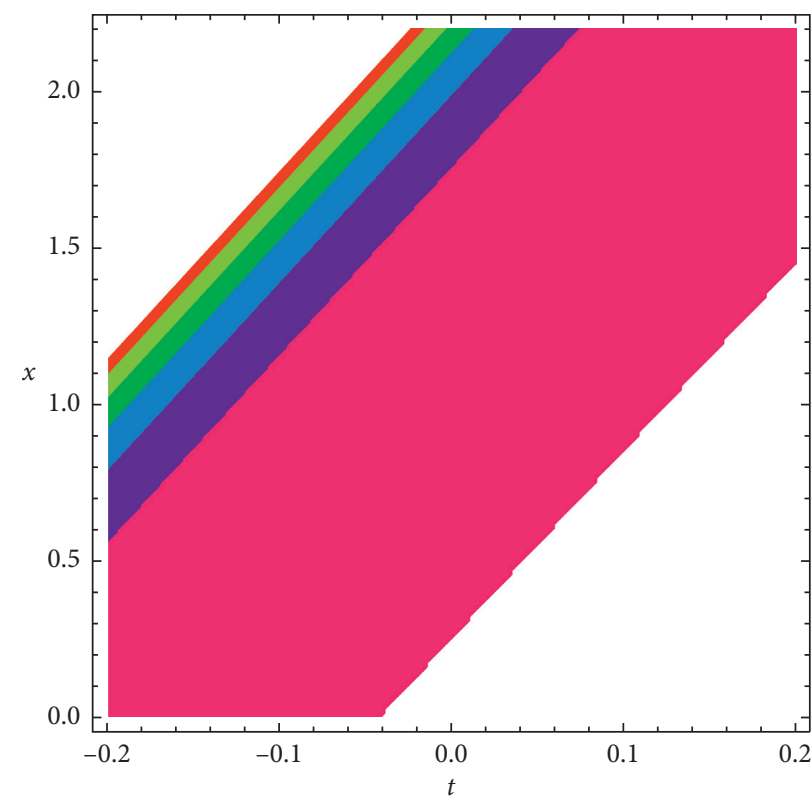

(c)

FIgURE 2: Solitary wave in three different forms of equation (31).

follow-up, we fix the value of $y$ to characterize these solutions and the interpretation is based on three different types of representations (three- and two-dimensional charts and contour plot). In the following steps, the physical interpretation of the represented figures is discussed:

(i) Figure 1 shows the bright solitary for (18) in the three-dimensional plot (a) to illustrate the perspective view of the solution, the two-dimensional plot (b) to present the wave propagation pattern of the wave along $x$ - axis, and the contour plot (c) to explain the overhead view of the solution when $\alpha=$ $-1, a_{0}=4, a_{-1}=0, a_{-2}=-3, c=72, \delta=2, \lambda=0, \mu=4$, and $\rho=3$

(ii) Figure 2 shows the dark solitary for (31) in the three-dimensional plot (a) to illustrate the perspective view of the solution, the two-dimensional plot (b) to present the wave propagation pattern of the wave along the $x$-axis, and the contour plot (c) to explain the overhead view of the solution when 


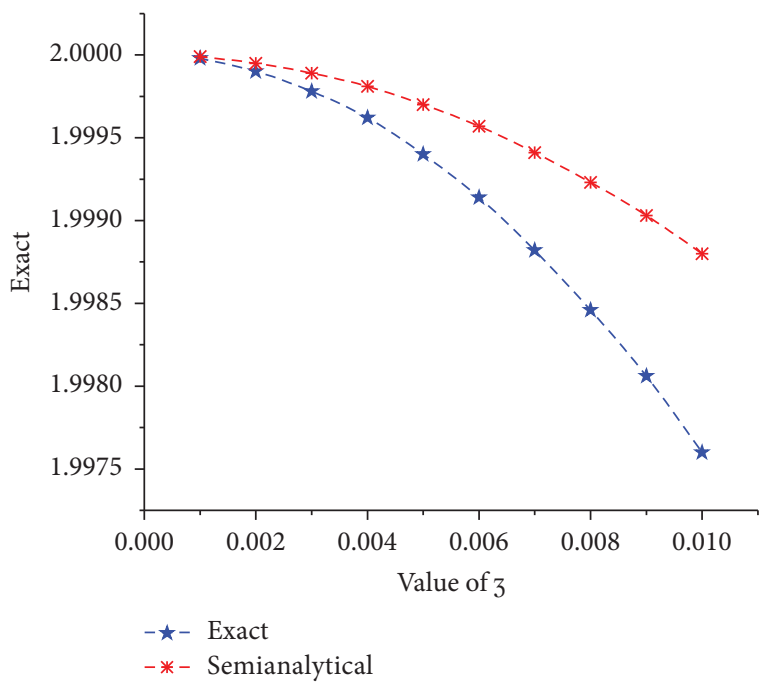

(a)

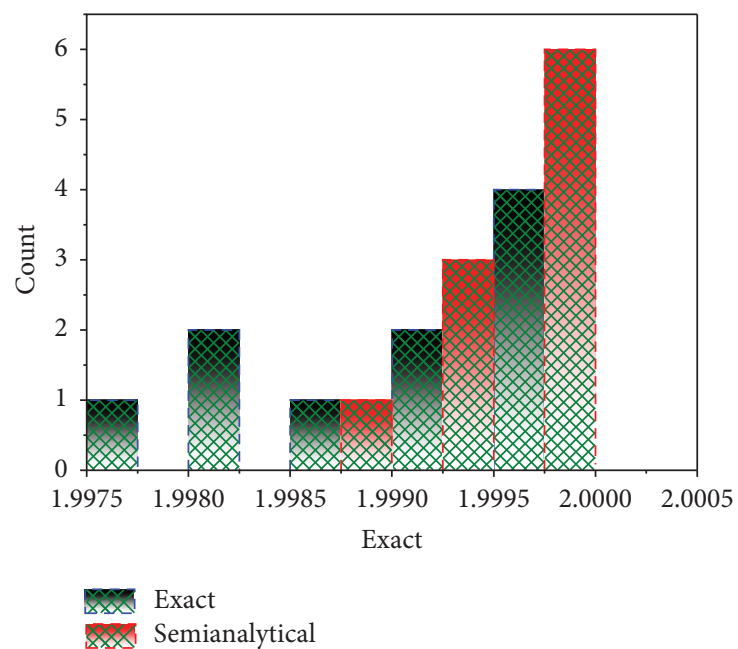

(b)

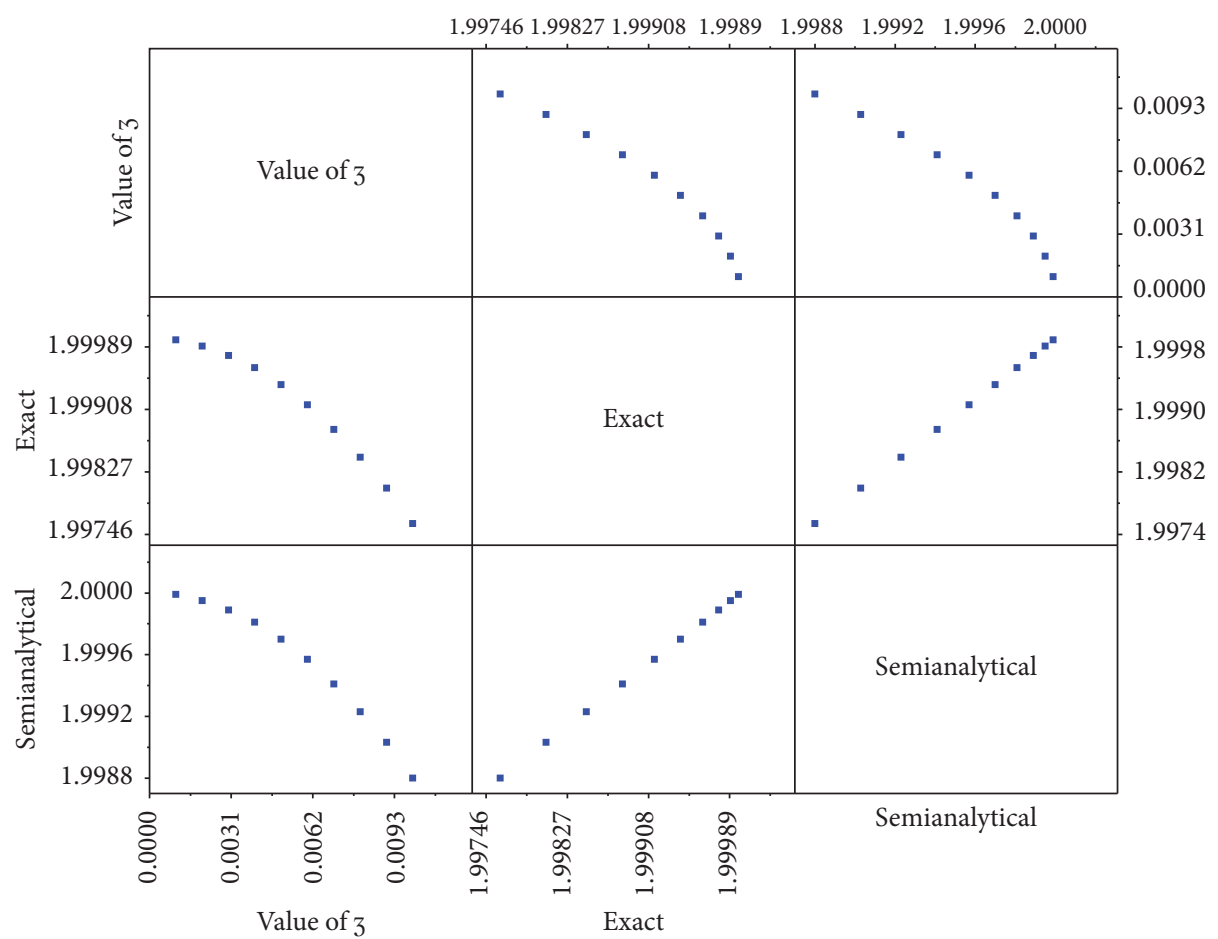

(c)

FIgURE 3: Exact and numerical solutions of equation (10) according to the obtained analytical solution via the extended simplest equation method.

$a_{0}=1, a_{1}=0, a_{2}=-(3 / 2), b_{1}=0, b_{2}=0, c=9, \delta=$ 4 , and $\rho=-(3 / 2)$

(iii) Figure 3 illustrates the exact and semianalytical obtained solutions, respectively, by the extended simplest equation method and Adomian decomposition method (iv) Figure 4 illustrates the exact and semianalytical obtained solutions, respectively, by the sech-tanh expansion method and Adomian decomposition method

(v) Figure 5 illustrates the exact and numerical obtained solutions, respectively, via the sech-tanh expansion method and cubic B-spline scheme 


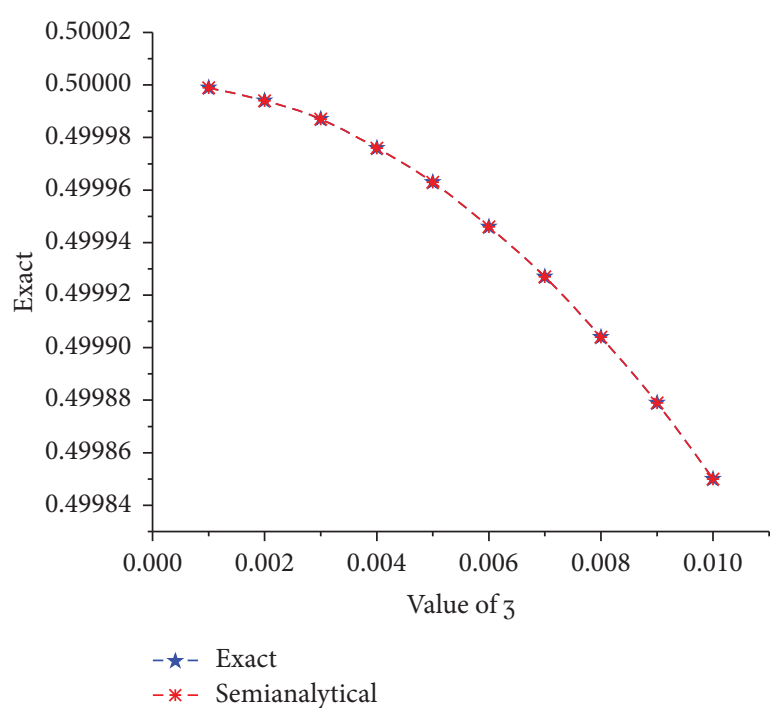

(a)

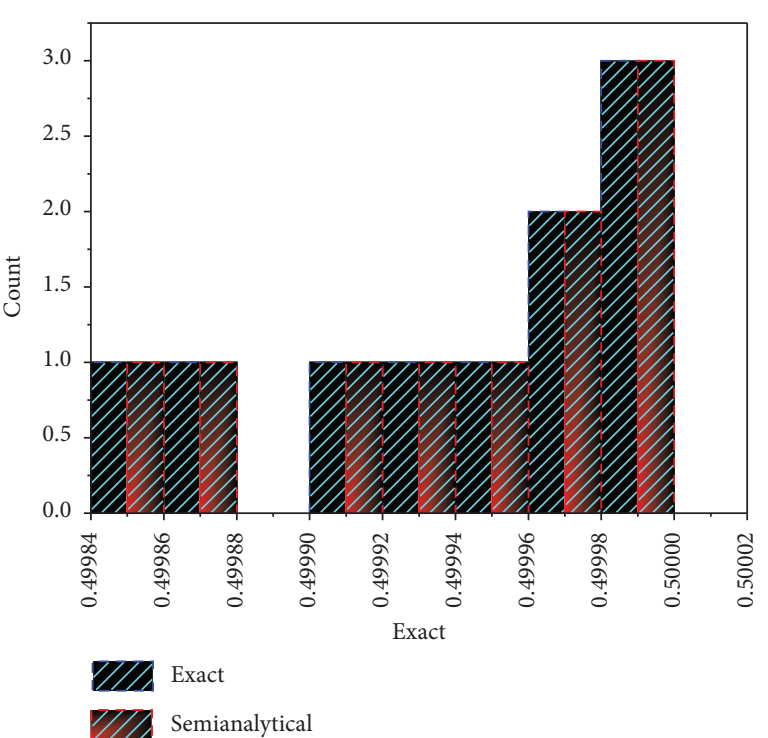

(b)

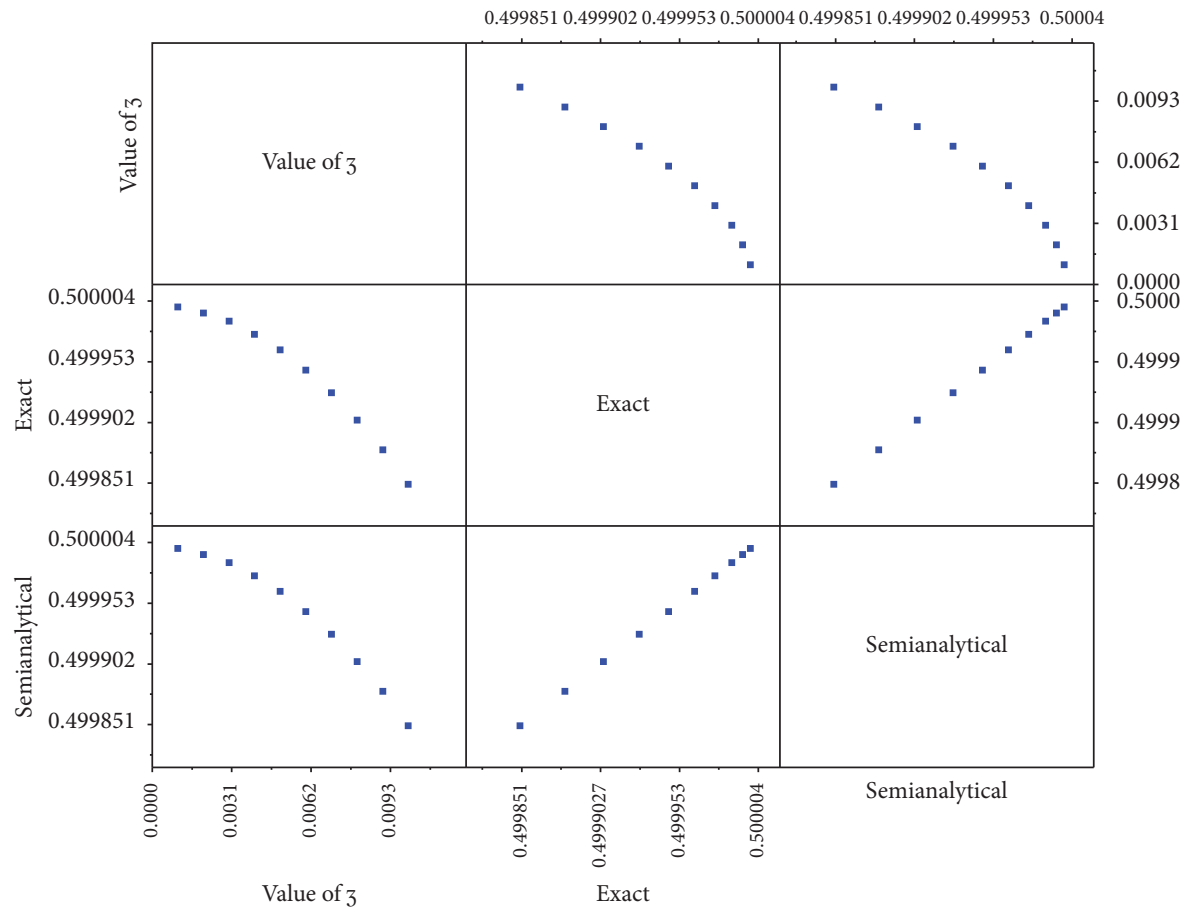

(c)

FIGURE 4: Exact and numerical solutions of equation (10) according to the obtained analytical solution via the sech-tanh expansion method.

(vi) Figure 6 illustrates the exact and numerical obtained solutions, respectively, via the sech-tanh expansion method and cubic B-spline scheme
Now, we shall show the accuracy of our obtained solution and explain the comparison between the two employed analytical schemes: 


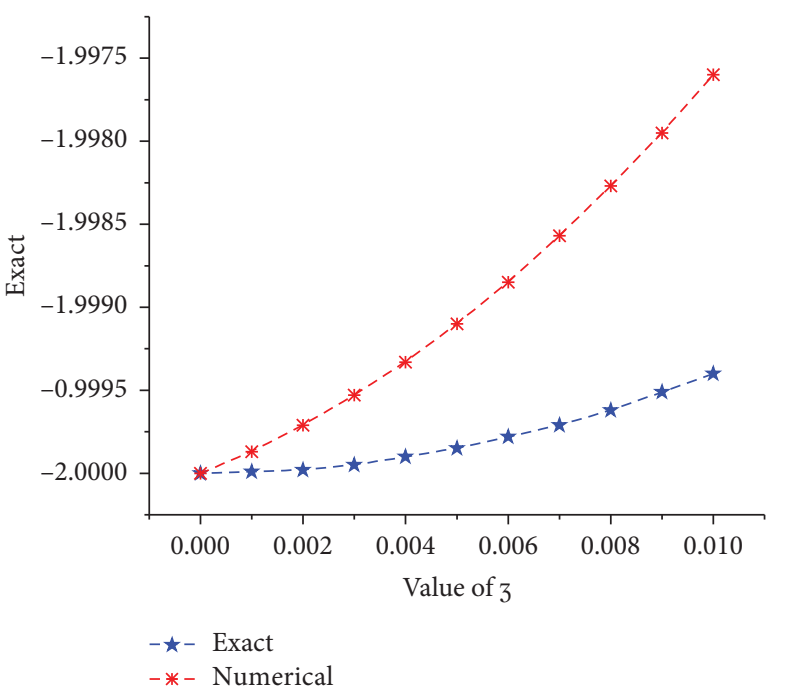

(a)

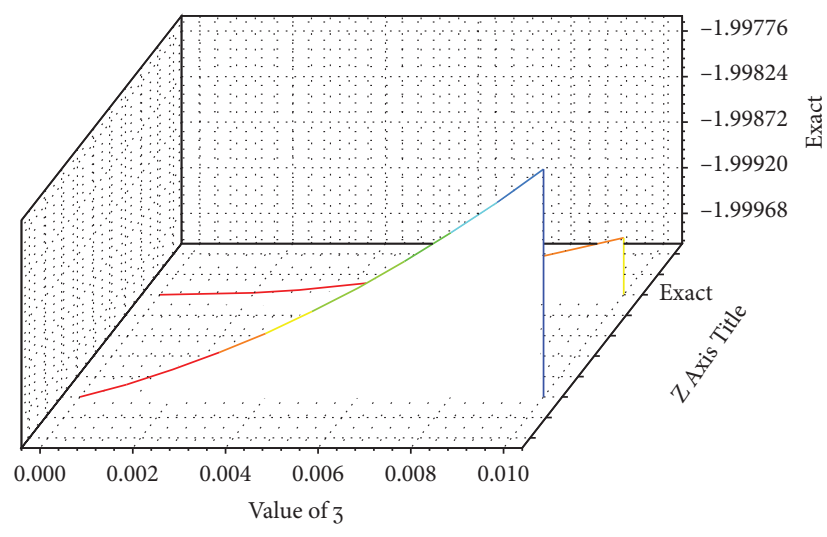

(b)

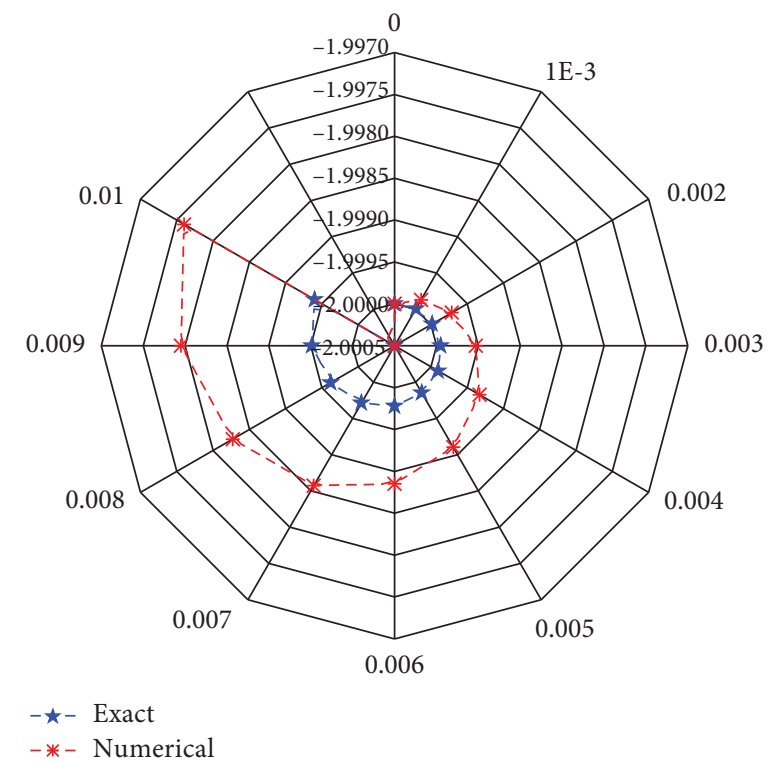

(c)

FIGURE 5: Exact and numerical solutions of equation (10) according to the obtained analytical solution via the extended simplest equation method.

(vii) Tables 1 and 2 show calculated values of the exact, semianalytical, and numerical solutions with different values of 3 . These values show the accuracy of the obtained analytical solutions via the sechtanh expansion method over the obtained analytical solutions via the extended simplest equation method where the absolute values of error in the sech-tanh method is smaller that those obtained by the extended simplest equation method. Figure 7 explains the absolute value of error in 1 and 2 . 


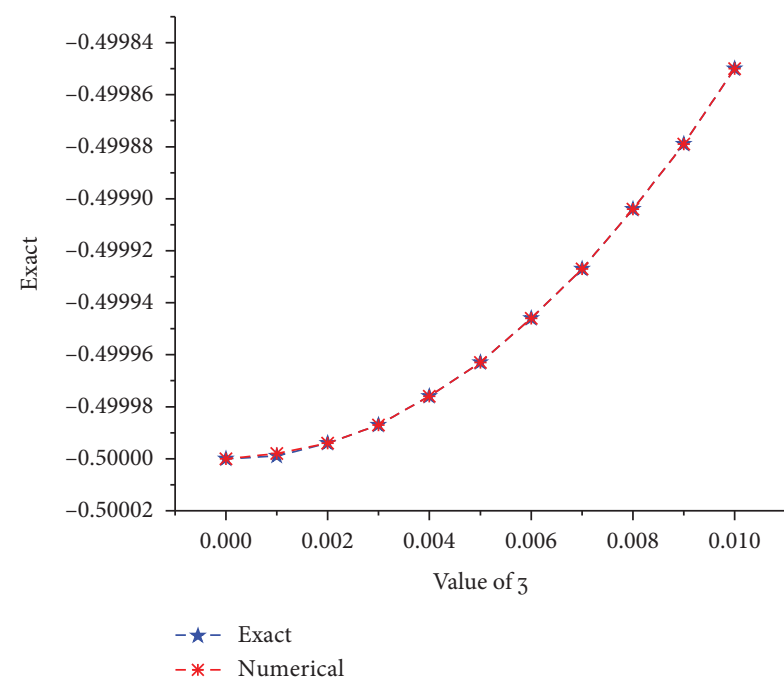

(a)

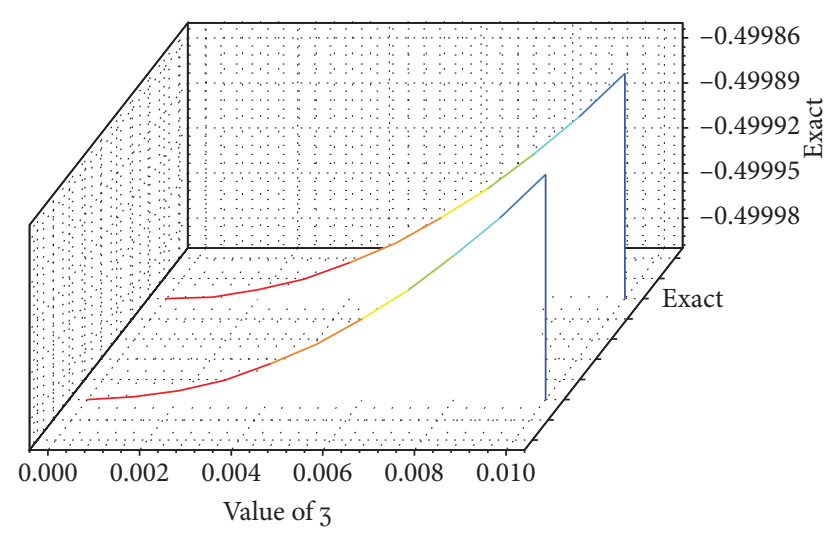

(b)

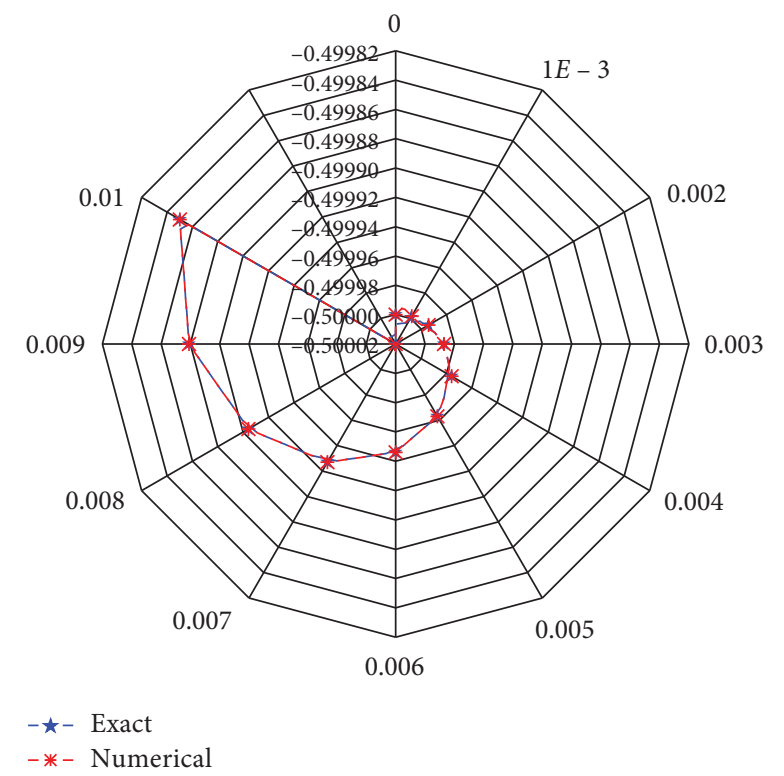

(c)

FIGURE 6: Exact and numerical solutions of equation (10) according to the obtained analytical solution via the sech-tanh expansion method.

TABLE 1: Exact, semianalytical, and absolute values of error with different values of 3 with respect to the obtained solution via extended simplest equation method (18) and sech-tanh method (31) via the Adomian decomposition method.

\begin{tabular}{lcccccc}
\hline & Ext. Sim Eq. method & & \multicolumn{2}{c}{ Sech-tanh method } & \multicolumn{2}{c}{ Absolute error } \\
Value of 3 & Exact & Approximate & Exact & Approximate & First scheme & Second scheme \\
\hline 0.001 & 1.99998 & 1.99999 & 0.499999 & 0.499999 & $1.19999 E-05$ & $4.99711 \times 10^{-13}$ \\
0.002 & 1.9999 & 1.99995 & 0.499994 & 0.499994 & 0.000047999 & $7.99988 \times 10^{-12}$ \\
0.003 & 1.99978 & 1.99989 & 0.499987 & 0.499987 & 0.000107995 & $4.05003 \times 10^{-11}$ \\
0.004 & 1.99962 & 1.99981 & 0.499976 & 0.499976 & 0.000191984 & $1.28001 \times 10^{-10}$ \\
0.005 & 1.9994 & 1.9997 & 0.499963 & 0.499963 & 0.00029996 & $3.12502 \times 10^{-10}$ \\
0.006 & 1.99914 & 1.99957 & 0.499946 & 0.499946 & 0.000431917 & $6.48006 \times 10^{-10}$ \\
0.007 & 1.99882 & 1.99941 & 0.499927 & 0.499927 & 0.000587846 & $1.20052 \times 10^{-9}$ \\
0.008 & 1.99846 & 1.99923 & 0.499904 & 0.499904 & 0.000767738 & $2.04804 \times 10^{-9}$ \\
0.009 & 1.99806 & 1.99903 & 0.499879 & 0.499879 & 0.00097158 & $3.28057 \times 10^{-9}$ \\
0.01 & 1.9976 & 1.9988 & 0.49985 & 0.49985 & 0.00119936 & $5.00013 \times 10^{-9}$ \\
\hline
\end{tabular}


TABLE 2: Exact, numerical, and absolute value of error with different value of 3 with respect to the obtained solution via the extended simplest equation method (18) and sech-tanh method (31) via the cubic B-spline scheme.

\begin{tabular}{|c|c|c|c|c|c|c|}
\hline \multirow{2}{*}{ Value of 3} & \multicolumn{2}{|c|}{ Ext. Sim Eq. Method } & \multicolumn{2}{|c|}{ Sech-tanh method } & \multicolumn{2}{|c|}{ Absolute error } \\
\hline & Exact & Numerical & Exact & Numerical & First scheme & Second scheme \\
\hline 0 & -2 & -2 & -0.5 & -0.5 & 0 & 0 \\
\hline 0.001 & -1.99999 & -1.99987 & -0.499999 & -0.499998 & 0.00012596 & $8.99958 \times 10^{-12}$ \\
\hline 0.002 & -1.99998 & -1.99971 & -0.499994 & -0.499994 & 0.000263919 & $1.59986 \times 10^{-11}$ \\
\hline 0.003 & -1.99995 & -1.99953 & -0.499987 & -0.499987 & 0.000413876 & $2.09974 \times 10^{-11}$ \\
\hline 0.004 & -1.9999 & -1.99933 & -0.499976 & -0.499976 & 0.000575829 & $2.39971 \times 10^{-11}$ \\
\hline 0.005 & -1.99985 & -1.9991 & -0.499963 & -0.499963 & 0.000749778 & $2.49963 \times 10^{-11}$ \\
\hline 0.006 & -1.99978 & -1.99885 & -0.499946 & -0.499946 & 0.000935721 & $2.39959 \times 10^{-11}$ \\
\hline 0.007 & -1.99971 & -1.99857 & -0.499927 & -0.499927 & 0.00113366 & $2.09953 \times 10^{-11}$ \\
\hline 0.008 & -1.99962 & -1.99827 & -0.499904 & -0.499904 & 0.00134358 & $1.59955 \times 10^{-11}$ \\
\hline 0.009 & -1.99951 & -1.99795 & -0.499879 & -0.499879 & 0.0015655 & $8.99764 \times 10^{-12}$ \\
\hline 0.01 & -1.9994 & -1.9976 & -0.49985 & -0.49985 & 0.0017994 & 0 \\
\hline
\end{tabular}

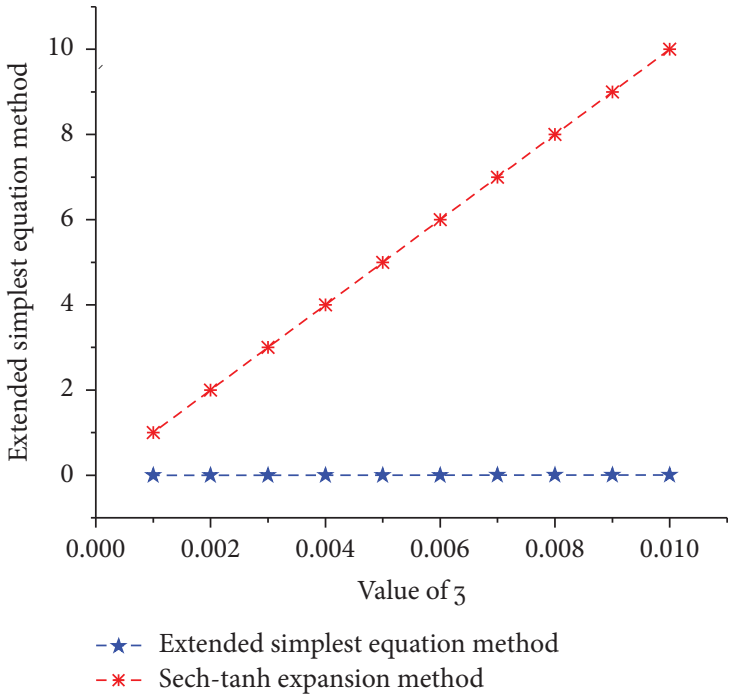

(a)

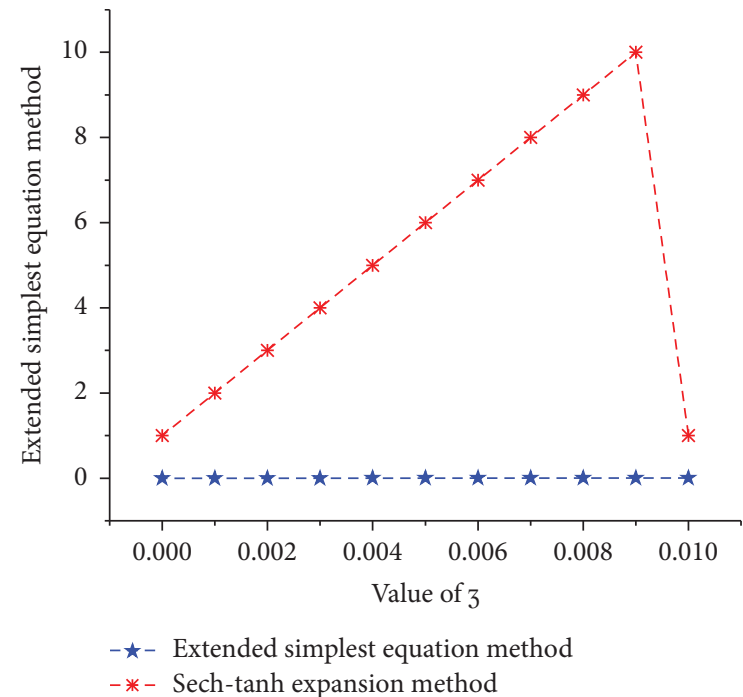

(b)

FIgURE 7: Absolute value of error via the Adomian decomposition method (a) and cubic B-spline scheme (b) based on the shown value in Tables 1 and 2 .

\section{Conclusion}

In this paper, the extended simplest equation and sechtanh expansion methods have been successfully implemented on the $(2+1)$-dimensional integrable SKdV model with Miura transform. Moreover, the stability properties of the solutions have also been tackled. The Adomian decomposition method and cubic B-spline scheme have also employed to investigate the semianalytical and numerical solutions, and the two show the accuracy of the obtained analytical solutions. The rigor of the obtained solutions that have been obtained by the sech-tanh expansion method has been discussed. The solutions were represented by allowing a physical interpretation and better interpretation of their properties. In summary, this paper studied the SKdV and found relevant solutions that provide new interpretations of the realworld phenomena.

\section{Data Availability}

Data sharing is not applicable for this article as no datasets were generated or analyzed during the current study.

\section{Conflicts of Interest}

The authors declare that there are no conflicts of interest.

\section{Authors' Contributions}

All authors conceived the study, participated in its design and coordination, drafted the manuscript, participated in the sequence alignment, and read and approved the final manuscript.

\section{Acknowledgments}

The authors extend their appreciation to the Deanship of Scientific Research at King Khalid University, Abha, KSA, 
for funding this work through the research group under grant number R.G.P-1/151/40.

\section{References}

[1] A. Seadawy, "Stability analysis of traveling wave solutions for generalized coupled nonlinear $\mathrm{KdV}$ equations," Applied Mathematics \& Information Sciences, vol. 10, no. 1, pp. 209214, 2016.

[2] M. S. Osman and A.-M. Wazwaz, "An efficient algorithm to construct multi-soliton rational solutions of the $(2+1)$-dimensional KdV equation with variable coefficients," Applied Mathematics and Computation, vol. 321, pp. 282-289, 2018.

[3] A.-M. Wazwaz and S. A. El-Tantawy, "A new integrable $(\$ \$ 3+1 \$ \$ 3+1)$-dimensional KdV-like model with its multiple-soliton solutions," Nonlinear Dynamics, vol. 83, no. 3, pp. 1529-1534, 2016.

[4] C. B. Jackson, C. Østerlund, G. Mugar, K. D. Hassman, and K. Crowston, "Motivations for sustained participation in crowdsourcing: case studies of citizen science on the role of talk," in Proceedings of the 2015 48th Hawaii International Conference on System Sciences, pp. 1624-1634, IEEE, Kauai, HI, USA, January 2015.

[5] A. H. Bhrawy, E. H. Doha, S. S. Ezz-Eldien, and M. A. Abdelkawy, "A numerical technique based on the shifted legendre polynomials for solving the time-fractional coupled KdV equations," Calcolo, vol. 53, no. 1, pp. 1-17, 2016.

[6] A. R. Seadawy, "The generalized nonlinear higher order of $\mathrm{KdV}$ equations from the higher order nonlinear Schrödinger equation and its solutions," Optik, vol. 139, pp. 31-43, 2017.

[7] A. El-Ajou, O. A. Arqub, and S. Momani, "Approximate analytical solution of the nonlinear fractional KdV-Burgers equation: a new iterative algorithm," Journal of Computational Physics, vol. 293, pp. 81-95, 2015.

[8] S. Lou and F. Huang, "Alice-Bob physics: coherent solutions of nonlocal KdV systems," Scientific Reports, vol. 7, no. 1, p. 869, 2017.

[9] W.-Q. Hu and S.-L. Jia, "General propagation lattice Boltzmann model for variable-coefficient non-isospectral $\mathrm{KdV}$ equation," Applied Mathematics Letters, vol. 91, pp. 61-67, 2019.

[10] I. M. Krichever and S. P. Novikov, "Holomorphic bundles over algebraic curves and non-linear equations," Russian Mathematical Surveys, vol. 35, no. 6, pp. 53-79, 1980.

[11] J. Weiss, “The Painlevé property for partial differential equations. II: Bäcklund transformation, Lax pairs, and the Schwarzian derivative," Journal of Mathematical Physics, vol. 24, no. 6, pp. 1405-1413, 1983.

[12] J. Weiss, "The Painlevé property and Bäcklund transformations for the sequence of Boussinesq equations," Journal of Mathematical Physics, vol. 26, no. 2, pp. 258-269, 1985.

[13] K. Toda and S.-J. Yu, "The investigation into the SchwarzKorteweg-de Vries equation and the Schwarz derivative in $(2+1)$ dimensions," Journal of Mathematical Physics, vol. 41, no. 7, pp. 4747-4751, 2000.

[14] M. S. Bruzón, M. L. Gandarias, C. Muriel, J. Ramírez, and F. R. Romero, "Traveling-wave solutions of the Schwarz-Korteweg-de Vries equation in 2+1 dimensions and the Ablowitz-Kaup-Newell-Segur equation through symmetry reductions," Theoretical and Mathematical Physics, vol. 137, no. 1, pp. 1378-1389, 2003.

[15] J. Ramírez, J. L. Romero, M. S. Bruzón, and M. L. Gandarias, "Multiple solutions for the Schwarzian Korteweg-de Vries equation in $(2+1)$ dimensions," Chaos, Solitons \& Fractals, vol. 32, no. 2, pp. 682-693, 2007.

[16] N. A. Kudryashov and A. Pickering, "Rational solutions for Schwarzian integrable hierarchies," Journal of Physics A: Mathematical and General, vol. 31, no. 47, pp. 9505-9518, 1998.

[17] A. Nakamura, "The Miura transform and the existence of an infinite number of conservation laws of the cylindrical KdV equation," Physics Letters A, vol. 82, no. 3, pp. 111-112, 1981.

[18] C. E. Kenig and Y. Martel, "Global well-posedness in the energy space for a modified KP II equation via the Miura transform," Transactions of the American Mathematical Society, vol. 358, no. 6, pp. 2447-2488, 2006.

[19] J. Mas and E. Ramos, "The constrained KP hierarchy and the generalised Miura transformation," Physics Letters B, vol. 351, no. 1-3, pp. 194-199, 1995.

[20] M. Senthil Velan and M. Lakshmanan, "Lie symmetries, KacMoody-Virasoro algebras and integrability of certain $(2+1)$ dimensional nonlinear evolution equations," Journal of Nonlinear Mathematical Physics, vol. 5, no. 2, pp. 190-211, 1998.

[21] H. Ma and Y. Bai, "New solutions of the Schwarz-Kortewegde Vries equation in 2+1 dimensions with the Gauge transformation," International Journal of Nonlinear Science, vol. 17, no. 1, pp. 41-46, 2014.

[22] M. Khater, R. Attia, and D. Lu, "Modified auxiliary equation method versus three nonlinear fractional biological models in present explicit wave solutions," Mathematical and Computational Applications, vol. 24, no. 1, 2019.

[23] M. M. Khater, D. Lu, and R. A. Attia, "Dispersive long wave of nonlinear fractional $\mathrm{Wu}$-Zhang system via a modified auxiliary equation method," AIP Advances, vol. 9, no. 2, p. 25003, 2019.

[24] R. Attia, D. Lu, and M. Khater, "Chaos and relativistic energymomentum of the nonlinear time fractional Duffing equation," Mathematical and Computational Applications, vol. 24, no. 1, p. 10, 2019.

[25] H. M. Baskonus, D. A. Koç, and H. Bulut, "Dark and new travelling wave solutions to the nonlinear evolution equation," Optik, vol. 127, no. 19, pp. 8043-8055, 2016.

[26] F. Dusunceli, "New exponential and complex traveling wave solutions to the Konopelchenko-Dubrovsky model," Advances in Mathematical Physics, vol. 2019, Article ID 7801247, 9 pages, 2019.

[27] Z. Odibat, "An optimized decomposition method for nonlinear ordinary and partial differential equations," Physica A: Statistical Mechanics and Its Applications, vol. 541, p. 123323, 2020.

[28] H. O. Bakodah, A. A. Al Qarni, M. A. Banaja, Q. Zhou, S. P. Moshokoa, and A. Biswas, "Bright and dark Thirring optical solitons with improved Adomian decomposition method," Optik, vol. 130, pp. 1115-1123, 2017.

[29] A. T. Ali, M. M. A. Khater, R. A. M. Attia, A.-H. Abdel-Aty, and $\mathrm{D}$. Lu, "Abundant numerical and analytical solutions of the generalized formula of Hirota-Satsuma coupled KdV system," Chaos, Solitons \& Fractals, vol. 131, p. 109473, 2020.

[30] M. M. Khater, C. Park, A.-H. Abdel-Aty, R. A. Attia, and D. Lu, "On new computational and numerical solutions of the modified Zakharov-Kuznetsov equation arising in electrical engineering," Alexandria Engineering Journal, vol. 59, no. 3, pp. 1099-1105, 2020. 